\title{
X-ray absorption spectroscopy study of cobalt mononitride thin films
}

\author{
Mukul Gupta $^{1}$ (1) $\cdot$ Yogesh Kumar $^{1} \cdot$ Akhil Tayal $^{2} \cdot$ Nidhi Pandey $^{1} \cdot$ Wolfgang Caliebe ${ }^{2} \cdot$ Jochen Stahn $^{3}$
}

Received: 1 August 2019 / Accepted: 27 November 2019 / Published online: 7 December 2019

(c) Springer Nature Switzerland AG 2019

\begin{abstract}
We present a comprehensive study to resolve the debate about the structure of cobalt mononitride (CoN). In contradiction with theoretical predictions, experimentally CoN has been claimed to synthesize in a rock-salt (RS) and in a zincblende (ZB)-type structure under ambient pressure and temperature. Utilizing X-ray absorption near-edge spectroscopy (XANES) at Co and N K-edges and extended X-ray absorption fine structure (EXAFS) at Co K-edge, we investigated the structure of $\mathrm{CoN}$ in detail. The presence of a strong pre-edge feature at the $\mathrm{Co} K$-edge and the absence of $\mathrm{t}_{2}$ feature at the $\mathrm{N} \mathrm{K}$-edge indicate towards the tetrahedral coordination that is expected in the ZB-type structure of CoN. Theoretical simulations of XANES spectra unambiguously confirm the ZB-type structure in CoN. Also from EXAFS data, we found that $\mathrm{Co}-\mathrm{N}$ and $\mathrm{Co}-\mathrm{Co}$ bond distances match well with ZB-CoN. Magnetic properties of CoN were examined using polarized neutron reflectivity and bulk magnetization measurements. It was found that $\mathrm{CoN}$ sample exhibits a paramagnetic behaviour as expected in ZB-CoN. Obtained results clearly demonstrate that $\mathrm{CoN}$ indeed crystalizes in the ZB-type structure, and the possibility of the RS-type CoN can be ruled out under ambient pressure and temperature. It is anticipated that the information about the structure of $\mathrm{CoN}$ can be utilized for its usage in emerging areas such as low-cost catalyst in oxygen and hydrogen evaluation reactions, high capacity anode in ion batteries and in photovoltaic applications.
\end{abstract}

Keywords Cobalt nitride $\cdot$ Cobalt mononitride $\cdot$ X-ray absorption spectroscopy $\cdot$ Transition metal nitrides

\section{Introduction}

Transition metal mononitrides (TMMN) of $3 d$ TM are an interesting class of compounds known to exhibit several interesting properties such as refractory nature, high electrical conductivity and chemical stability, applications as catalysis, energy storage and conversion and more recently in plasmonics [1-5]. Most of these properties are exhibited by early TMMN, viz. ScN, TiN, VN and CrN. In recent years, late TMMN, in particular $\mathrm{CoN}$, have also been explored as an emerging material in renewable and sustainable energy. Though initial experimental research works on CoN were more curiosity driven [6-9], in recent years, it has been shown that $\mathrm{CoN}$ can be used as a high capacity anode in Li-ion batteries [10,11], as a non-aqueous supercapacitor [12], as a low-cost catalyst for oxygen and hydrogen evolution reaction (OER and HER) required for electrocatalysis and water splitting [13-17] and for mesoscopic and perovskite solar cells [18].

Therefore, it is essential to have a deeper understanding of the structural and the magnetic properties of cobalt nitrides (Co-N). Co-N compounds are known to form in a variety of compositions and structures. Major $\mathrm{Co}-\mathrm{N}$ phases that have been identified are: $\mathrm{Co}_{16} \mathrm{~N}_{2}(\mathrm{bct})$, $\mathrm{Co}_{4} \mathrm{~N}(\mathrm{fcc}), \mathrm{Co}_{3} \mathrm{~N}(\mathrm{hcp}), \mathrm{Co}_{2} \mathrm{~N}$ (orthorhombic), $\mathrm{CoN}(\mathrm{fcc}), \mathrm{CoN}_{2}$ (marcasite-type). Among these, the $\mathrm{Co}_{16} \mathrm{~N}_{2}$ phase is being

Electronic supplementary material The online version of this article (https://doi.org/10.1007/s42452-019-1808-2) contains supplementary material, which is available to authorized users.

\footnotetext{
$\triangle$ Mukul Gupta, mgupta@csr.res.in | 1 UGC-DAE Consortium for Scientific Research, University Campus, Khandwa Road, Indore 452 001, India. ${ }^{2}$ Deutsches Elektronen-Synchrotron, Notkestraße 85, 22607 Hamburg, Germany. ${ }^{3}$ Laboratory for Neutron Scattering and Imaging, Paul Scherrer Institut (PSI), CH-5232, Villigen, Switzerland.
} 
explored recently [24], $\mathrm{Co}_{2} \mathrm{~N}$ and $\mathrm{Co}_{3} \mathrm{~N}$ have been studied by several co-workers [25-29]. The $\mathrm{N}$-rich $\mathrm{CoN}_{2}$ phase was recently synthesized under high pressure high temperature (30 GPa, $1600 \mathrm{~K}$ ) [30]. In this work, we will focus on the cobalt mononitride (CoN) phase only.

Though early TMMN are known to crystalize in a common rock-salt (RS)-type structure [31], late TMMN such as FeN and CoN exhibit a different behaviour as they are debated to crystalize in a zinc-blende (ZB) and a RS-type structure [21]. In the ZB-type structure, both FeN and CoN are non-magnetic. The RS-type FeN is expected to possess a nonzero magnetic moment, but in the RS-type CoN, a finite magnetic moment is expected only under a lattice expansion [21]. Among the late TMMN, FeN is the most studied compound yet its structural and magnetic ground state is still debated $[32,33]$.

From recent experimental works, it can be observed that FeN crystalizes in the ZB-type structure only (under ambient pressure and temperature) and the existence of the RS-type FeN has been questioned in several recent works [34-36]. Though theoretical works predict both ZBand RS-type structures for FeN and CoN, but under ambient temperature and pressure, the ZB-type structure is preferred [21]. The energy minima in the total energy-volume plots is lower in ZB, as compared to that in RS CoN [21, 22, 37-40]. Under high pressure, a phase transition from ZB- to RS-type phase is expected at about $50 \mathrm{GPa}$ in FeN [33] and about $40 \mathrm{GPa}$ in CoN [22].

From a brief review of $\mathrm{CoN}$ compounds (see Table 1), it can be seen that the assignment to the RS- or ZB-type structure and associated lattice parameter (LP) is random. In early works, Dumont and Kron [19] obtained CoN by thermal decomposition of the cobalt amide- $\mathrm{Co}\left(\mathrm{NH}_{2}\right.$ )$_{3}$ and found RS-type structure with a LP $=4.27 \AA$ from $X$-ray diffraction (XRD) measurements. Subsequently, Taylor et al. [23] prepared CoN by thermal decomposition of cobalt ammine azide [Co $\left.\left(\mathrm{NH}_{3}\right)_{6}\right]\left(\mathrm{N}_{3}\right)_{3}$ and reported $\mathrm{ZB}$ type $\mathrm{CoN}$ with $\mathrm{LP}=4.28 \AA$. More recently, in a pioneering work seeking the structural and magnetic properties of CoN, Suzuki et al. [6] deposited CoN thin films on a $\mathrm{Cu}$ substrate using reactive dc sputtering of Co with a mixture of $\mathrm{Ar}+\mathrm{N}_{2}$ gases. The film was stripped from the substrate and treated with nitric acid and annealed at $410 \mathrm{~K}$ for $6 \mathrm{~h}$ in vacuum. Thereafter, powdered samples were measured using XRD. By comparing the experimental data with theory for the ZB- and RS-type structures, they confirmed ZB-type CoN with LP $=4.297$ A. From detailed low-temperature measurements, they reported that $\mathrm{CoN}$ exhibits a Pauli paramagnetic character [6].

In recent years, Das et al. performed nitridation of cobalt tetraoxide $\mathrm{Co}_{3} \mathrm{O}_{4}$ and $\mathrm{NiCO}_{2} \mathrm{O}_{4}$ in the presence of $\mathrm{NH}_{3}+\mathrm{N}_{2}$ and claimed RS-type $\mathrm{CoN}$ in Ni-doped CoN nanoparticles with $\mathrm{LP}=4.295 \AA$ from XRD and Co $K$-edge XANES measurements [10, 11]. Liu et al. [20] prepared epitaxial $\mathrm{CoN}$ thin films on $\mathrm{SrTiO}_{3}$ and sapphire substrates using laser ablation method, and from XRD measurements they found that the LP of their CoN samples was $4.27 \AA$. . They also performed low-temperature magnetization measurements and claimed RS-type CoN due to appearance of a para-to-antiferromagnetic transition $\approx 310 \mathrm{~K}$. Very recently, Kang et al. [18] prepared CoN samples by rf sputtering on fluorine-doped tin oxide (FTO)-coated glass and indium tin oxide (ITO)/polyethylene naphthalate (PEN) substrates. They found a strong pre-edge feature in Co $K$-edge XANES

Table 1 Lattice parameter (LP) and structure of CoN thin films grown using different methods and using different calculation methods [fullpotential local density approximation(FP-LDA), generalized gradient approximation by Perdew-Burke-Ernzerhof (GGA-PBE)]

\begin{tabular}{|c|c|c|c|c|}
\hline Methodology & $\operatorname{LP}(\AA ̊)$ & Str. type & Str. & Reference technique \\
\hline Decomposition of cobalt amide $\mathrm{Co}\left(\mathrm{NH}_{2}\right)_{3}$ & 4.27 & RS & XRD & [19] \\
\hline Nitridation of cobalt tetraoxide $\mathrm{Co}_{3} \mathrm{O}_{4}$ & 4.295 & RS & XRD & {$[10]$} \\
\hline Nitridation of $\mathrm{NiCO}_{2} \mathrm{O}_{4}$ & 4.294 & RS & XRD + XANES (Co K-edge) & [11] \\
\hline Laser ablation of $\mathrm{Co}+\mathrm{N}_{2}$ & 4.27 & RS & XRD + Magnetization & {$[20]$} \\
\hline Theoretical (FP-LDA) & 3.972 & RS & Theory & {$[21]$} \\
\hline Theoretical (GGA-PBE) & 4.010 & RS & Theory & {$[22]$} \\
\hline $\begin{array}{l}\text { Decomposition of cobalt ammine azide [Co } \\
\left.\left(\mathrm{NH}_{3}\right)_{6}\right]\left(\mathrm{N}_{3}\right)_{3}\end{array}$ & 4.28 & ZB & XRD & [23] \\
\hline Dc sputtering of Co by $\left(\mathrm{Ar}+\mathrm{N}_{2}\right)$ & 4.297 & ZB & XRD & {$[6]$} \\
\hline Rf sputtering of Co by $\mathrm{N}_{2}$ & 4.33 & ZB & XRD + EXAFS(Co K-edge) & [18] \\
\hline Theoretical (FP-LDA) & 4.276 & ZB & Theory & {$[21]$} \\
\hline Theoretical (GGA-PBE) & 4.26 & ZB & Theory & {$[22]$} \\
\hline Dc sputtering of Co by $\mathrm{N}_{2}$ & 4.32 & ZB & $\begin{array}{l}\text { XRD + XANES(Co, N K-edge)+ } \\
\text { EXAFS(Co } K \text {-edge) + magnetization }\end{array}$ & This work \\
\hline
\end{tabular}

Here, RS denotes rock-salt and ZB to zinc-blende-type structure (str.) of CoN 
spectra indicating that $\mathrm{Co}$ atom has tetrahedral symmetry which is expected in ZB-type CoN. A summary of these experimental works is shown in Table 1 and compared with recent theoretical predictions. CoN samples have also been prepared in some other works, but structural information (RS or ZB) was not provided [7-9, 14, 41].

It is to be noted that in most of the experimental works performed so far, the structure of $\mathrm{CoN}$ was identified using XRD only. The LP of CoN in RS- and ZB-type structures was found to be almost similar around $4.29 \pm 0.02 \AA$, the only difference between the $Z B$ and RS structures is arrangements of $\mathrm{N}$ atoms - tetrahedral in $\mathrm{ZB}$ and octahedral in RS. Therefore, the difference in the structure factor leads to a variation in relative intensities, e.g. in ZB-type structure, the intensity of (111) reflection would be highest, whereas in RS-type structure (200) reflection would become most intense. In the case of powdered samples, this analogy holds good (as demonstrated by Suzuki et al. [6]) but in the case of epitaxial or polycrystalline films with a preferred orientation such analogy about relative intensity would not work and alternative ways should be explored to establish the structure of $\mathrm{CoN}$ thin films. This can be easily achieved utilizing $X$-ray absorption fine structure (XAFS) techniques-XANES and EXAFS measurements at a synchrotron radiation source [42].

In the present work, we performed reactive dcMS (dc magnetron sputtering) of a Co target using $\mathrm{N}_{2}$ alone as the process gas (no $\mathrm{Ar}$ ) at room temperature $(300 \mathrm{~K}$ ) to get polycrystalline CoN thin films on $\mathrm{Si}(100)$ and amorphous quartz substrates. The synthesis of $\mathrm{CoN}$ using chemical route involves expensive and toxic cobalt-based compounds [11]. Reactive sputtering on the other hand is a clean, reproducible and industry-friendly method to synthesize CoN from the metal Co target. We employed various techniques to investigate the structural and magnetic properties of $\mathrm{CoN}$ thin films. Moreover, to probe the local structure in $\mathrm{CoN}$ thin films, XAFS measurements performed both at $\mathrm{Co}$ and $\mathrm{N}$ K-edge are also presented.

\section{Experimental and theoretical procedures}

Thin films of CoN were deposited using a home-made sputtering system, and a Co ( $\phi 2$ in., purity $99.95 \%$ ) target was sputtered from a magnetron source (Angstrom Sciences) in $\mathrm{N}_{2}$ (purity $99.9995 \%$ ) environments. The sputtering power was fixed at about $45 \mathrm{~W}\left(2.2 \mathrm{Wcm}^{-2}\right)$, and samples were deposited at ambient temperature (300 K) without any intentional heating. As shown recently, that $\mathrm{N}$ diffuse out from $\mathrm{CoN}$ even when the substrate temperature is raised marginally, it is essential to keep substrates at room temperature to grow the desired $\mathrm{CoN}$ phase [43]. With a base pressure of about $1 \times 10^{-7} \mathrm{hPa}$, the pressure during deposition was about $5 \times 10^{-3} \mathrm{hPa}$ due to the flow of $\mathrm{N}_{2}$ gas at $50 \mathrm{sccm}$. Samples were deposited on a high-quality amorphous quartz and Si (100) (both from Matsurf-Tech) substrates simultaneously. Substrates were continuously rotated at a speed of $30 \mathrm{rpm}$, and film thickness of about $100 \mathrm{~nm}$ was achieved in $1 \mathrm{~h}$. Prior to deposition of final samples, several samples were deposited to achieve optimum growth conditions to attain the CoN phase [44]. Samples of size typically $5 \mathrm{~cm}^{2}$ were deposited on Si and amorphous quartz substrates simultaneously and small samples (about $1 \mathrm{~cm}^{2}$ ) cut from these were subject to different measurements that are reported in this work.

The structure of samples deposited on amorphous quartz substrates was determined using X-ray diffraction (XRD) using a standard diffractometer (Bruker D8 Advance) and using $\mathrm{Cu}-K_{\alpha} \mathrm{X}$-rays in $\theta-2 \theta$ mode. X-ray absorption near-edge spectroscopy (XANES) measurements at $\mathrm{N} K$-edge were performed at the soft X-ray absorption spectroscopy (SXAS) beamline BL01 of Indus 2 synchrotron radiation (SR) source at RRCAT, Indore, India [45]. They were performed in the total electron yield (TEY) mode under ultra-high vacuum conditions.

XANES and extended $X$-ray absorption fine structure (EXAFS) measurements at Co $K$-edge were carried out under ambient conditions at the P64 beamline [46] of Petra III, DESY, Hamburg, Germany. These measurements were performed in the fluorescence geometry, and the integrated fluorescence $\left(l_{f}\right)$ from the sample was measured with a passivated implanted planar silicon detector. The upstream beam intensity $\left(I_{0}\right)$ was measured with a gas ionization detector filled with pure nitrogen. The normalized absorption was obtained using the expression $I_{f} / I_{0}$. The incident flux on the sample was about $10^{12}$ photons/sec, and absorption in the upstream ionization chamber was $8 \%$ at $7000 \mathrm{eV}$, which is in the range where the beamline detector operates linearly. The dark current is properly offset, and no counts in the $I_{0}$ or $I_{f}$ were observed in the absence of X-ray beam. Energy calibration of a $\mathrm{Si}(111)$ monochromator was performed using an iron foil. The first inflection point in the absorption spectrum of iron foil was assigned to $7112 \mathrm{eV}$. Frequent measurement on iron foil was carried out to check the monochromator calibration, which was found to be stable during the entire experiment with nearly identical edge positions found for all the measurements. For the XANES and EXAFS measurements, samples of $10 \mathrm{~mm} \times 10$ $\mathrm{mm}$ were sliced from the $50 \mathrm{~mm} \times 50 \mathrm{~mm}$ sample. The beam size on the samples was about $1 \mathrm{~mm} \times 1 \mathrm{~mm}$. Radiation damage on the samples was checked by measuring the spectrum at a different spot. Since no variation in the near-edge feature and EXAFS oscillation could be 
observed, any damage due to radiation can be ruled out. Multiple XAFS scans (six/twelve) were performed on each sample at a fixed beam spot, and the total measurement time was about 30 minutes. Lousy data points occurred in the spectrum due to the monochromator glitches were removed, and the polynomial interpolation was used to connect the stripped region.

Standard procedure was employed to carry out preedge and post-edge background subtraction [47]. The normalization of the data was performed by simulating a Victoreen polynomial function obtained from the regression of the absorption spectrum in the pre-edge region. The polynomial was then extrapolated to the end of the absorption spectrum and subtracted from the data. For the extraction of the EXAFS signal, an atomic-like background was calculated in the post-edge region using a polynomial spline of third order with three knots. The positions of knots were then optimized to remove the low-frequency background between 0-1 A. The EXAFS fits were performed in the $k$-space in the range 3-13 $\AA^{-1}$, and five shells were used to fit the spectrum in the $R$-range between 1.1-4.5 $\AA$ covering first three shells of ZnS-type CoN, respectively, for Co-N (1.87 $\AA), \mathrm{Co}-\mathrm{Co}(3.03 \AA)$, and Co-Co $(4.25 \AA ̊)$. The number of independent fitting parameters for this range is about 21. The EXAFS fitting was performed to derive metrical parameters for each shell, such as atomic pair distance $(R)$, root-mean-square relative displacement $(\sigma)$, coordination number $(N)$, and relative change in $E_{0}\left(\Delta E_{0}\right)$. For the five shells, it gives a total number of parameters 20 , among which the parameter $\Delta E_{0}$ for the first shell is varied independently, and its value for all the shell was kept constrained to vary between \pm 1 relative to the value obtained from the fits for the first shell. In the absence of a bulk CoN sample, the value of passive electron reduction factor $\left(S_{0}^{2}\right)$ cannot be estimated, which usually varies between $0.8-0.9$. Therefore, it was fixed at an average value of 0.9 , which may give some errors in the estimation of the actual coordination number. However, it does not influence the results obtained in this work. The EXAFS fitting and background subtraction of the data was performed using the software written by Conradson et al. [48]. The photoelectron backscattering amplitude and phase-shift for different $\mathrm{Co}-\mathrm{N}$ and $\mathrm{Co}-\mathrm{Co}$ shells on ZB-type $\mathrm{CoN}$ and $\mathrm{CO}_{2} \mathrm{~N}$ phases were theoretically calculated using the FEFF9 code [49].

XANES spectra at Co $\mathrm{K}$-edge and $\mathrm{N} \mathrm{K}$-edge were theoretically calculated using real-space multiple-scattering formalism using FDMNES code [50]. The lattice parameters for ZB- and RS-type structure of CoN were kept at $4.32 \AA$ which is obtained from the XRD. Self-consistent calculations were performed to determine the Fermi energy. Hedin-Lundqvist exchange correlation potential was used for the simulation. The convolution of XANES spectra was performed using theoretically determined core hole width. The simulation is carried out for the atomic cluster with the radius of $9 \AA$.

Magnetization measurements were performed using a Quantum Design made SQUID-VSM (S-VSM) magnetometer and polarized neutron reflectivity (PNR) at AMOR, SINQ, PSI, Switzerland, in time of flight mode using Selene optics [51,52]. Thermal stability of samples was studied by using an ultra-high vacuum (base pressure $1 \times 10^{-9} \mathrm{hPa}$ ) rapid thermal annealing system, and they were annealed for $1 \mathrm{~h}$ at each temperature. XRD and $\mathrm{N} K$-edge XANES measurements were carried out in annealed samples.

\section{Results and discussion}

XRD measurements carried out on pristine and vacuum annealed samples are shown in Fig. 1. In the pristine sample, a broad peak appears around $35.95^{\circ}$ and it can be assigned to CoN (111) (JCPDS No. 830831), yielding lattice parameter $(L P)=4.32( \pm 0.01) \AA$. The $L P$ of our sample has been compared to $\mathrm{CoN}$ samples grown using different methods and also with theoretical calculations as shown in Table 1. Here, it can be seen that the theoretical value LP in RS- and ZB-type structure is around 4.0 and $4.27 \AA$, respectively, and experimental values $4.29( \pm 0.03) \AA$ are much more closer to the ZB-type rather than to the RStype CoN. Therefore, assignment to RS-type structure on the basis of LP appears dubious.

The crystallite size calculated using Scherrer formula comes out to be $\approx 8 \mathrm{~nm}$. It may be noted that samples

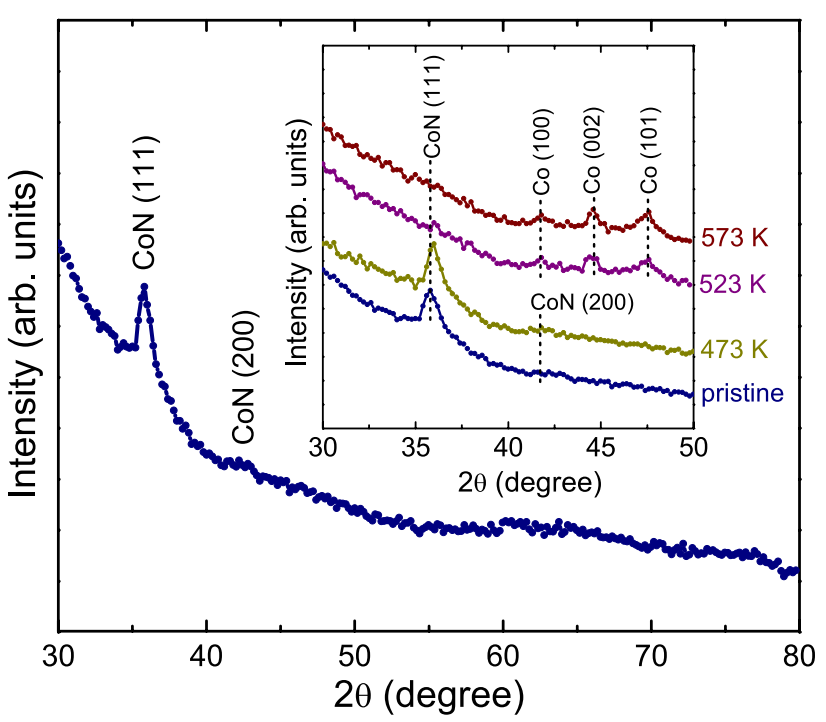

Fig. 1 X-ray diffraction pattern CoN thin film grown at $300 \mathrm{~K}$ (pristine). The inset compares XRD patterns of pristine and vacuum annealed samples. They were annealed at different temperatures for $1 \mathrm{~h}$ in a vacuum furnace 
were deposited at ambient temperature and their thickness was only about $100 \mathrm{~nm}$; this may lead to the formation of a nanocrystalline structure in the sample. Further, the peak profile appears somewhat asymmetric which may be due to some defects or vacancies. Similar defects or vacancies were also observed in FeN samples and characterized using Mössbauer spectroscopy measurements [34, 36]. Unlike FeN, the absence of Mössbauer nuclei in CoN makes it difficult to probe such defects or vacancies. After annealing the pristine sample at $473 \mathrm{~K}$, the peak shifts marginally to $2 \theta \approx 36.10^{\circ}$, reducing LP to $4.30( \pm 0.01) \AA$. Such reduction in LP can be due to relaxation of quenched-in voids that get generated during thin film growth. After annealing at $523 \mathrm{~K}$, the peak corresponding to $\mathrm{CoN}$ (111) almost disappears and peaks corresponding to hcp Co start to appear and they get more intense when annealing temperature was further raised to $573 \mathrm{~K}$. Thermal stability of our $\mathrm{CoN}$ thin films is in agreement with previous works $[6,41,53]$.

The thermal stability behaviour can be understood comparing the enthalpy of formation energy $\left(\Delta H_{f}^{\circ}\right)$ of $\mathrm{CoN}$ together with other metal nitrides. For several nitrides $\Delta H_{f}^{\circ}$ (in eV/atom) is negative, -0.5 for FeN [54], -1.2 for $\mathrm{CrN}$ [55], -3.5 for TiN [56] but for $\mathrm{CoN}$ it is $\approx 0$ [57]. Therefore, early TMMN such as $\mathrm{CrN}$ and TiN are easy to form but difficult to decompose but $\mathrm{CoN}$ would be difficult to synthesize but gets decomposed easily upon heating. The decomposition temperature for early TMMN exceeds $1000 \mathrm{~K}[58]$, whereas for $\mathrm{FeN}$ it is about $600 \mathrm{~K}[36,59]$ and for CoN even less (450-500 K) [6]. In fact as shown in a recent work, when $\mathrm{Co}-\mathrm{N}$ films are deposited using reactive sputtering at $523 \mathrm{~K}$, resulting films resemble pure Co rather than any Co-N phase [53]. Though poor thermal stability of $\mathrm{CoN}$ is a concern, it is a boon as well for its usage in catalytic reactions [60].

In order to further explore the local structure, we did $\mathrm{N} K$-edge XANES and Co $K$-edge XANES and EXAFS measurements. Figure 2 shows $\mathrm{N} K$-edge XANES of $\mathrm{CoN}$ thin films in the pristine state and after annealing at $523 \mathrm{~K}$. In the pristine state, prominent $\mathrm{N} K$-edge can be seen and features present there are marked as $a, b, c$ and $d$. Features $a$ and $b$ correspond to transition from $\mathrm{N} 1 s$ to hybridized states of $\mathrm{Co} 3 d$ and $\mathrm{N} 2 p$, and features $c$ and $d$ appear due to due to electronic transition from $\mathrm{N} 1 \mathrm{~s}$ to hybridized $\mathrm{N}$ $2 p$ and $\mathrm{Co} 4 s p$ states. The $\mathrm{N} \mathrm{K}$-edge features in our case are similar to those in ZB-FeN [59]. In a octahedral symmetry (RS) due to crystal-field splitting, the edge features split into two $-\mathrm{t}_{2 g}$ and $\mathrm{e}_{g}$ states, whereas as in tetrahedral (ZB) symmetry, the crystal-field splitting is reversed and they are denoted by e and $t_{2}$ (the gerade $-g$ label is removed as symmetry is non-centrosymmetric). Such features have been clearly resolved in the case of early TMMN such as TiN [61] and CrN [55, 62]. The absence of any significant

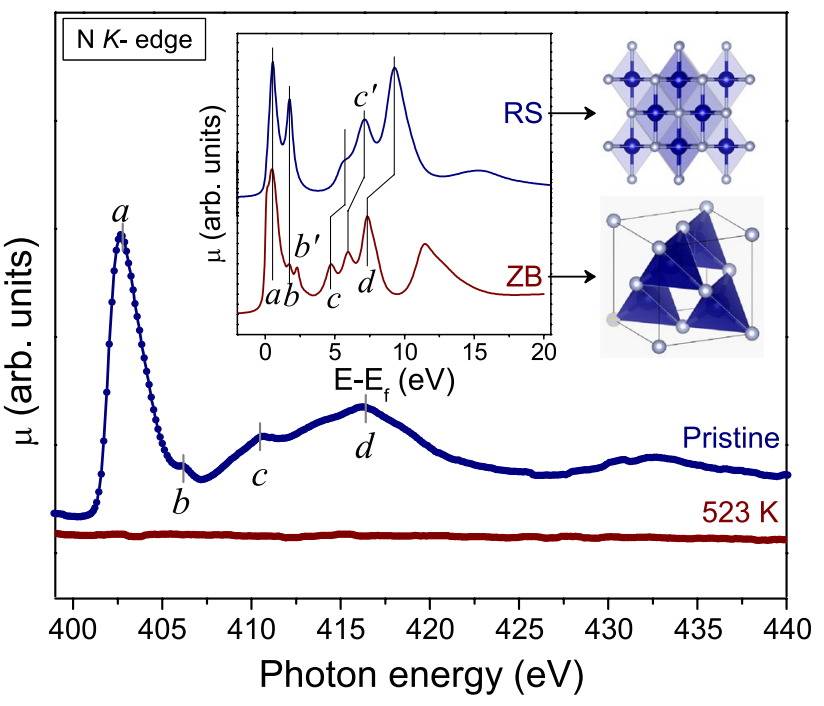

Fig. $2 \mathrm{~N} \mathrm{~K}$-edge XANES of CoN thin film in the pristine state and after annealing at $523 \mathrm{~K}$. The inset shows simulated N K-edge XANES of CoN in RS- and ZB-type structures along with their schematic representations

splitting around feature $a$ is a clear indication of tetrahedral coordination of $C o$ atoms surrounding $N$ atoms. It may be noted that though $\mathrm{N} K$-edge features have been studied in several works for early TMMN [63], for CoN they have not been reported hitherto. After annealing at $523 \mathrm{~K}$, all N features disappear which can happen only if $\mathrm{N}$ is diffusing out from CoN leaving behind Co.

$\mathrm{N} K$-edge was also generated using FDMNES code for CoN in ZB- and RS-type structures as shown in the inset of Fig. 2. Analogous to the experimental case, prominent features in simulated spectrum are also assigned as $a, b, c$ and $d$. It can be seen that the feature $b$ is more pronounced in RS and weaker in ZB-type CoN. Qualitatively, theoretically simulated $\mathrm{N} K$-edge features resemble with experiment, but their energy difference with respect to $a$ does not match. The separation between the first two features ( $a$ and $b$ ), i.e. $10 D q$, comes out to be about $1.5 \mathrm{eV}$ from simulations but experimentally, it is much larger at about $4 \mathrm{eV}$. Such difference between theory and experiments can also be observed in other nitrides, e.g. in $\mathrm{CrN}, \mathrm{VN}$ and $\mathrm{NbN}$ theoretical values of $10 \mathrm{Dq}$ are $0.8,1.1$ and $2 \mathrm{eV}[63,64]$, respectively, whereas experimental values were about $3 \mathrm{eV}$ for $\mathrm{CrN}[55,65], 2.7 \mathrm{eV}$ for VN [66] and $3.5 \mathrm{eV}$ for NbN [67]. Therefore, combing the resemblance of our $\mathrm{N} \mathrm{K}$-edge spectra in CoN together with that of ZB-FeN and to the simulated spectra for ZB-CoN, it appears that the structure of $\mathrm{CoN}$ in our samples is closer to ZB-type.

We also did Co $K$-edge XANES and EXAFS measurements to further confirm the structure of our sample as shown in Fig. 3. The presence of an intense pre-edge feature can be clearly seen in the near-edge region and is 


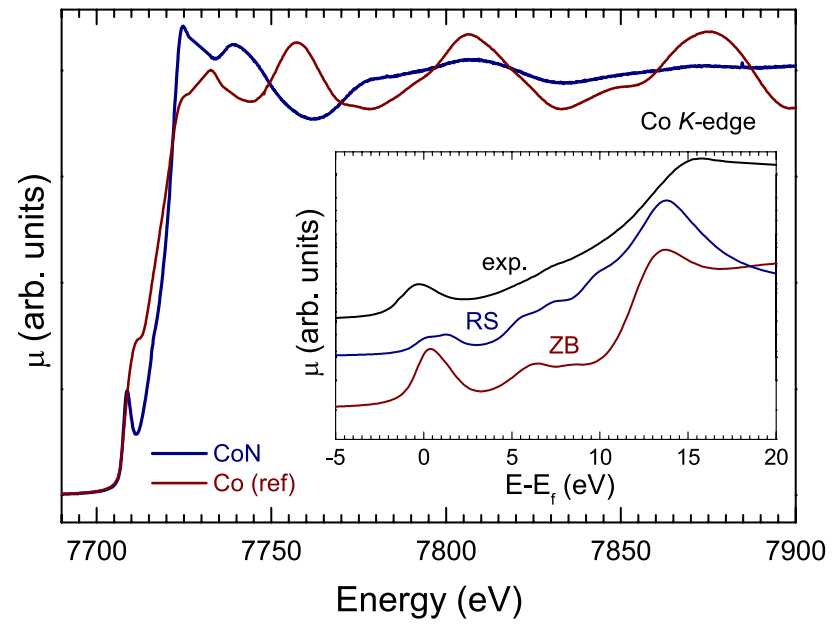

Fig. 3 Co $K$-edge XANES of CoN thin film sample in the pristine state compared with a bulk Co reference. The inset shows simulated Co K-edge XANES of CoN in RS- and ZB-type structures. Here, Co (ref.) corresponds to a Co foil with hcp structure

indicative of tetrahedral coordination expected in ZB-type CoN. The inset of Fig. 3 compares experimental XANES spectrum together with theoretically simulated spectra for CoN in RS- and ZB-type structures. Clearly, our experimental data resembles well with ZB-type CoN. It must be noted that the intensity of pre-edge features in the experimental spectrum is lower compared to the theoretically calculated spectra. The smearing of pre-edge features is the cumulative effect of broadening due to instrumental resolution, and nanocrystalline structure of the sample, as also observed in other metallic nanoparticles [68-70].

To get quantitative information, EXAFS pattern (of the same sample as shown in Fig. 3) was fitted and is shown in Fig. 4; and obtained metrical fitting parameters are listed in Table 2. At the starting model, the EXAFS fitting was performed with the first three shells in the ZB-type CoN. The value of $\sigma$ for the higher-order shells is constrained to vary between \pm 0.02 relative to the previous shell, and other parameters were kept free to vary except the $\Delta \mathrm{E}_{0}$ and the $S_{0}^{2}$ as discussed in the experimental section. In the ZB-type $\mathrm{CoN}, \mathrm{Co}$ atoms are tetrahedrally coordinated with $\mathrm{N}$ atoms. The bond lengths for $\mathrm{Co}-\mathrm{N}$ bond were found to be about $1.87 \AA$ and for the Co-Co bond it was about $3.0 \AA$ and $4.25 \AA$ for the second and third coordination shells. The first peak around $1.6 \AA$ in the FT spectra is attributed to the $\mathrm{Co}-\mathrm{N}$ shell, and the EXAFS fitting shows the $\mathrm{Co}-\mathrm{N}$ bond distance is about $1.87 \AA$. The FT peak between $2-3 \AA$ can be attributed to the Co-Co shell. However, it was observed that in this region, the FT peak is quite broad and slightly different on comparing it with the spectrum reported by Kang et al. [18] on relatively thick ZB-type CoN films. Further, it can be seen from Fig. 4 that the peak shoulder between 2-2.6 ̊̊ has a significantly higher amplitude. It

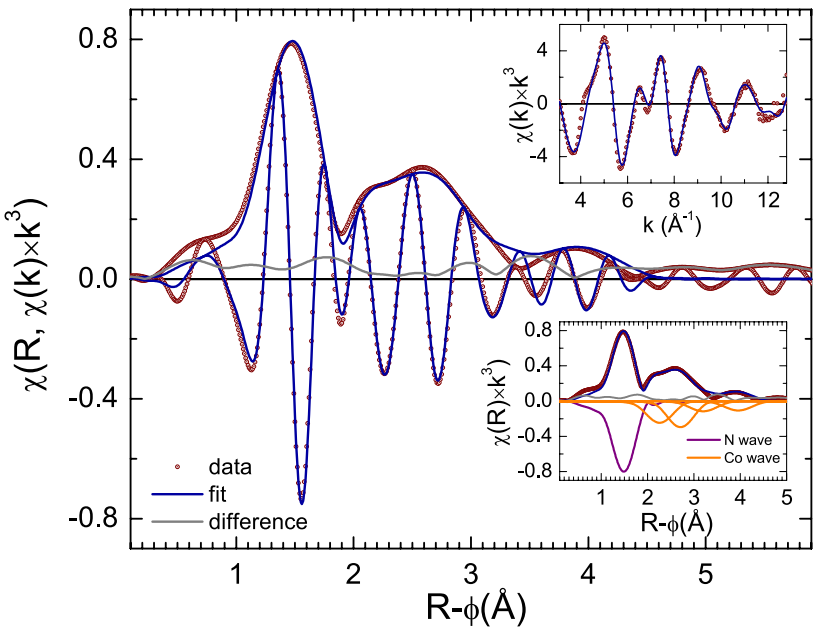

Fig. 4 Fourier-transform moduli of the Co K-edge EXAFS $(|\chi(R)|)$ and real component $(\operatorname{Re}[\chi(R)])$ of the data and fit. Lower inset shows individual components of the fit which are inverted for clarity. Upper inset shows $\chi(k) \times k^{3}$ data and fit. $R-\phi$ is phase-shiftuncorrected atomic pair distance

results in the presence of a significant residual component between 2-2.6 $\AA$, and 3-3.5 $\AA$. The inclusion of Co-Co shell at $2.57 \AA$ and $3.54 \AA$ significantly improves the quality of the fit. The amplitude and phases of additional shells were obtained by performing the FEFF calculation on $\mathrm{CO}_{2} \mathrm{~N}$ having Co-Co shells at similar distances. With this approach, the value of the coordination number for the higher Co-Co shells was found to be smaller relative to the bulk. It is interesting to note that the value of $\mathrm{N}$ for the first Co-N shell is slightly smaller compared with the ideal tetrahedral geometry. For lower Co-N phases or RS-type CoN, the value of $N$ is six due to octahedral geometry. Slightly lower coordination number from the ideal tetrahedral geometry can be attributed to the atomic defects and nanometresized particles [71].

Alternatively, we have also modelled the EXAFS fitting by constraining the $N$ for Co-Co shell of ZB-type structure to vary between \pm 0.5 relative to thrice the value of $N$ for the first Co-N shell and half the value of this shell, respectively, for Co-Co shells at $3.0 \AA$ and $4.25 \AA$ (see electronic supplementary malarial [72], fig. S1; table ST1). With this approach, we observed that the value of coordination number for higher $\mathrm{Co}-\mathrm{Co}$ shell comes close to the bulk; however, the value of $\sigma$ inflated to twice the value observed for the first $\mathrm{Co}-\mathrm{N}$ shell. Moreover, the coordination number for the additional shell at $3.54 \AA$ is found to be extremely small, and the shell distance at $2.57 \AA$ is shifted to $2.63 \AA$. A four shell fitting to the data excluding second extra shell was also tried (see electronic supplementary malarial [72] fig. S2; table ST1). This fitting approach indicates that a strong correlation exists between the EXAFS parameters, such as $N$ and $\sigma$. 
Table 2 EXAFS metrical parameters derived from nonlinear least-square fits for $\mathrm{CoN}$ thin films measured at Co K-edge

\begin{tabular}{llllll}
\hline Co $K$-edge & $\begin{array}{l}\text { Co-N } \\
\text { ZB-type }\end{array}$ & $\begin{array}{l}\text { Co-Co } \\
\text { ZB-type }\end{array}$ & $\begin{array}{l}\text { Co-Co } \\
\text { Extra }\end{array}$ & $\begin{array}{l}\text { Co-Co } \\
\text { Extra }\end{array}$ & $\begin{array}{l}\text { Co-Co } \\
\text { ZB-type }\end{array}$ \\
\hline$R(\AA)$ & $1.87 \pm 0.01$ & $3.04 \pm 0.02$ & $2.57 \pm 0.02$ & $3.54 \pm 0.02$ & $4.29 \pm 0.02$ \\
$N$ & $3.0 \pm 0.6$ & $1.2 \pm 0.3$ & $0.8 \pm 0.2$ & $1.3 \pm 0.4$ & $1.6 \pm 0.5$ \\
$\sigma$ & $0.06 \pm 0.01$ & $0.08 \pm 0.01$ & $0.09 \pm 0.01$ & $0.10 \pm 0.02$ & 0.10 \\
$\Delta E_{0}$ & 0.0 & $0.0 \pm 2.0$ & $0.0 \pm 3.0$ & $0.0 \pm 2.0$ & 0 \\
\hline
\end{tabular}

Here, $R$ is obtain atomic pair distance, $N$ is coordination number, $\sigma$ is root mean square displacement and $\Delta E_{0}$ energy is shift parameter. The parameters reported without error bars were not varied during the fitting
Therefore, additional fitting constraints are required to obtain the absolute value of the coordination number. Moreover, in the thin films, various parameters such as particle size distribution, defects and disorder can correlate with the EXAFS amplitude, and hence accurate estimation of coordination number is challenging [71, 73, 74].

The EXAFS fitting was also modelled by incorporating the higher-order cumulants for the additional shell at $2.57 \AA$ (see electronic supplementary malarial [72] fig. S3; table ST1) $[74,75]$. It was observed that the fitting improved significantly with the inclusion of the third cumulant. However, due to its correlation with the phase, the shell distance was found at $2.82 \AA$. The multiple EXAFS modelling suggests that the absolute value of the coordination number is difficult to obtain. Besides, the additional shell is highly disordered and may have an anharmonic distribution of atomic pair distance. Therefore, it cannot be attributed to any $\mathrm{Co}-\mathrm{N}$ phases. Such highly disordered Co-Co atomic pairs can be observed around the defects and vacancies sites. The local region around these defects can adopt the local structure of lower (than $\mathrm{CoN}$ ) concentration nitride phases to minimize the energy and can give similar pair distances.

The above analysis implies that the CoN crystallizes in the ZB-type structure. However, deposition condition can influence the defects and disorder in the sample. It can give distinct local structure; however they are localized in the proximity of defects sites. These findings corroborate with the XANES, XRD and magnetization results discussed as follows. The absence of additional XRD peaks suggests that the extra Co-Co shell is not the part of any crystalline domains and remains as a disordered or non-crystalline phase. Moreover, the magnetization measurements show absence of any magnetic component which ruled out the possibility of any lower (magnetic) $\mathrm{Co}-\mathrm{N}$ phases. It is to be emphasized that the use of pure nitrogen as the sputtering gas will preclude the formation of isolated metallic clusters of Co with 1-2 nm particle size. Such nanoparticle clusters will be highly reactive in the nitrogen plasma. Therefore, its presence in the sample can be ruled out.
Magnetization and the magnetic ground state of mononitrides of $\mathrm{Fe}$ and $\mathrm{Co}$, i.e. FeN and $\mathrm{CoN}$, have been a long-standing concern and have been often correlated with the structure. For FeN, it is anticipated that it will be paramagnetic (PM) in the ZB-type structure but would have some magnetic ordering, i.e. ferromagnetic (FM) or antiferromagnetic (AFM) in the RS-type structure [76]. However, CoN is expected to remain PM even in the RStype structure unless it is forced to adopt an expanded lattice $[21,76]$. Therefore, recent experimental results show a PM-to-AFM transition around $310 \mathrm{~K}$, and based on such transitions claiming the structure to be RS-type needs to be investigated further [20]. Anticipating the complexity of this issue, we not only used bulk magnetization methods (S-VSM) but polarized neutron reflectivity (PNR) technique as well to probe magnetism in our $\mathrm{CoN}$ samples. As a reference, a Co thin film sample that was prepared under identical conditions (but using $\mathrm{Ar}$ instead of $\mathrm{N}_{2}$ ) was also measured.

PNR is a well-known technique to probe magnetization and magnetic depth profiles of thin film sample. In this technique, substrate magnetization (often diamagnetic as in present case for Si substrate) does not interfere with sample magnetization. Neutrons are spin $\frac{1}{2}$ particles and possess quantization states that are aligned parallel or antiparallel (denoted by ' $+{ }^{\prime}$ or ' $-{ }^{\prime}$ ) with respect to the direction of applied external field $(H)$. A dipolar interaction takes places between neutron magnetic moment and the magnetic field generated by unpaired spins [77]. The interaction potential is given by $V_{m}=-\mu_{n} \cdot \mathbf{B}$, where magnetic induction $\mathbf{B}=H+4 \pi M, \mu_{n}$ is neutron magnetic moment and $M$ is sample magnetization[78].

The information about sample magnetization can be directly inferred by just observing the separation between spin-up $\left(R^{+}\right)$and spin-down $\left(R^{-}\right)$reflectivities at the critical value of wave vector transfer $\left(q_{c}\right)$, given by $q_{c}{ }^{ \pm}=\sqrt{\left(16 \pi N\left(b_{n} \pm b_{m}\right)\right.}$, where $N$ is the number density, $b_{n}$ and $b_{m}$ are the nuclear and magnetic scattering lengths for neutrons [79]. As can be seen from Fig. 5a, the PNR pattern of the reference Co thin film sample exhibits well-separated $\mathrm{R}^{+}$and $\mathrm{R}^{-}$at $q_{c}$, confirming the 
Fig. 5 Polarized neutron reflectivity (PNR) measurements of $\mathrm{CON}$ and $\mathrm{Co}$ thin film samples measured at $300 \mathrm{~K}$ (a). The inset of (a) compares the spin-asymmetry $\left(\mathrm{SA}=\left(\mathrm{R}^{+}-R^{-}\right.\right.$ )$\left./\left(R^{+}+R^{-}\right)\right)$of experimental PNR data of CoN and Co samples. The zero field cooled (ZFC) and field cooled (FC) at $\mathrm{H}=100$ Oe magnetization (M)-temperature $(\mathrm{T})$ plots of CoN thin film compared with $M$ of Co measured at $300 \mathrm{~K}(\mathbf{b})$. The inset of $\mathbf{b}$ compares $\mathrm{M}-\mathrm{H}$ loops of $\mathrm{CoN}$ and Co samples measured at $300 \mathrm{~K}$
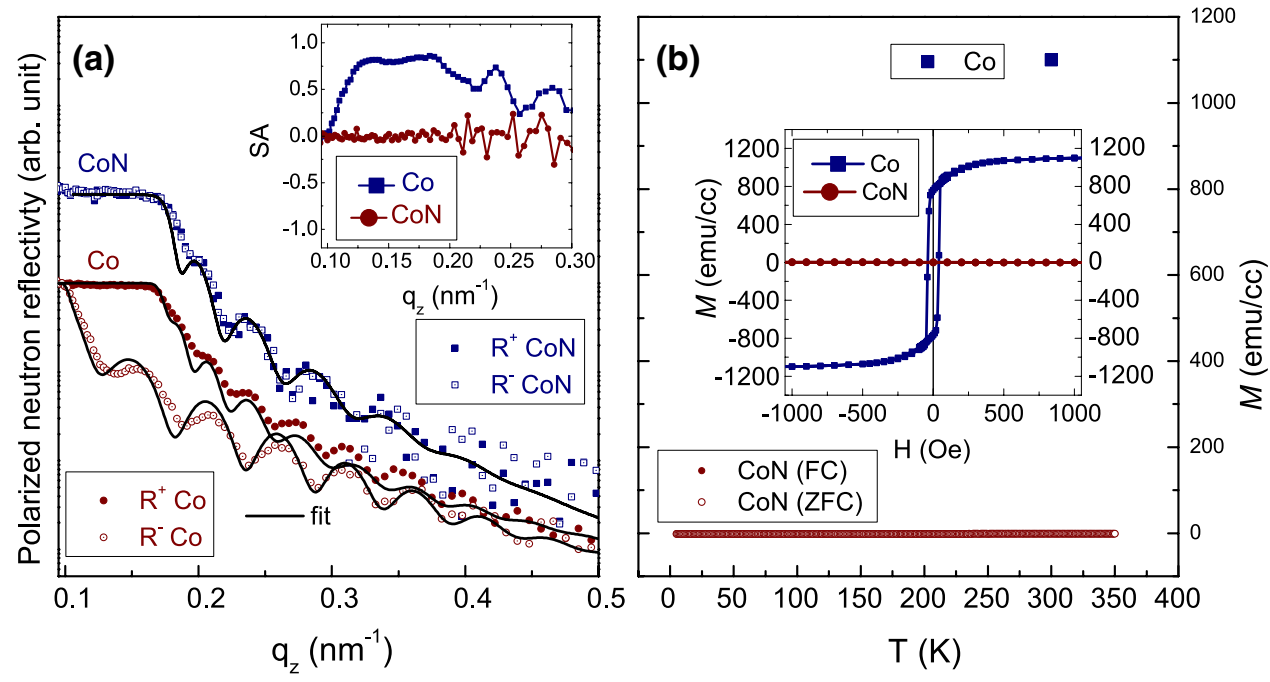

FM ordering in Co and yielding $M=1.6 \mu_{\mathrm{B}}$ per Co atom which is expected in bulk Co [41]. But such separation cannot be seen in the CoN sample. In the PM state, $b_{m}=$ 0 , and therefore, $R^{+}=R^{-}$. Thus, from our PNR measurements it can be directly seen that CoN sample adopts a $\mathrm{PM}$ state which is expected for CoN in ZB-type structure at $300 \mathrm{~K}$ [6]. This also becomes evident by comparing the spin-asymmetry $\left(\mathrm{SA}=\left(\mathrm{R}^{+}-R^{-}\right) /\left(\mathrm{R}^{+}+\mathrm{R}^{-}\right)\right)$of $\mathrm{CoN}$ and $\mathrm{Co}$ samples as shown in the inset of Fig. $5 \mathrm{a}$. For $\mathrm{CoN}$ since $\mathrm{R}^{+}=\mathrm{R}^{-}, \mathrm{SA} \approx 0$, whereas for Co large separation between $R^{+}$and $R^{-}$is large near the critical $q_{z}$ due to large ferromagnetic moment of $\mathrm{Co}$.

PNR patterns were fitted using SimulReflec [80] software and obtained fitting parameters are: thickness of CoN film $=95 \mathrm{~nm}$, roughness $\approx 1 \mathrm{~nm}$, film density $\approx 6 \mathrm{~g}$ $\mathrm{cm}^{-3}$ and $M=0$. The thickness, roughness and density of the film were also obtained from $X$-ray reflectivity measurements (not shown) and match well with PNR results.

Magnetization measurements were also performed using bulk magnetization method (S-VSM). The $M-H$ hysteresis loops (taken at $300 \mathrm{~K}$ ) for $\mathrm{CoN}$ and Co samples are compared in the inset of Fig. $5 \mathrm{~b}$. The saturation magnetization of Co sample comes out to be about $1100 \mathrm{emu} \mathrm{cm}^{-3}$ matching well with bulk Co, but for CoN, we can see that $M \approx 0$, which is in agreement with PNR results. We also did magnetization measurements by lowering the sample temperature down to $5 \mathrm{~K}$ under zero field cooled (ZFC) and field cooled (FC) conditions (at $100 \mathrm{Oe}$ ) and such M-T data are shown in Fig. $5 \mathrm{~b}$ and again compared with Co magnetization value obtained at $300 \mathrm{~K}$. As can be seen, the $M$ of CoN remains at almost zero both in FC and ZFC cases. Therefore, it can be concluded from our magnetization data that $\mathrm{CoN}$ sample exhibited a paramagnetic behaviour down to $5 \mathrm{~K}$.

\section{Conclusions}

The present study shows the systematic investigation of reactively sputtered CoN thin films grown at the ambient temperature. XRD analysis on the pristine film shows single diffraction peak that was attributed to the ZB-type CoN phase. The XRD peak was found to have an asymmetric shape which was assigned to the intrinsic defects introduced during the sputtering. Magnetization measurement does not show any signature of ferromagnetism. Experimental XANES spectra measured at $\mathrm{N} \mathrm{K}$-edge and $\mathrm{Co} \mathrm{K}$-edge were compared with the theoretically simulated spectra on the ZB- and RS-type CoN. It was observed that the near-edge features in the experimentally measured spectra are closely associated with the theoretically simulated spectra on ZB-type CoN. An intense pre-edge peak in the Co K-edge spectrum provides further evidence of tetrahedral coordination of Co in the sample. The local structure of Co was further investigated by performing the least-square fitting on the EXAFS data. The EXAFS fitting reveals that apart from the presence of $\mathrm{Co}-\mathrm{N}$ and $\mathrm{Co}-\mathrm{Co}$ atomic pairs similar to those found in the ZB-type $\mathrm{CoN}$, additional $\mathrm{Co}-\mathrm{Co}$ atomic pairs were observed. These extra $\mathrm{Co}-\mathrm{Co}$ atomic pairs are attributed to the defects in the sample around which the Co has adopted local structure similar to the lower nitride phases.

Acknowledgements Authors thank the Department of Science and Technology, India (SR/NM/Z-07/2015), for the financial support and Jawaharlal Nehru Centre for Advanced Scientific Research (JNCASR) for managing the project. A part of this work was performed at AMOR, Swiss Spallation Neutron Source, Paul Scherrer Institute, Villigen, Switzerland. We acknowledge technical help received from L. Behera, R. J. Choudhary for S-VSM, V. R. Reddy and A. Gome for XRR and A. Wadikar and R. Sah for N K-edge XANES measurements. 
We are thankful to V. Ganesan and A. K. Sinha for support and encouragement.

\section{Compliance with ethical standards}

Conflict of interest The authors declare that they have no conflict of interest.

\section{References}

1. Rasaki SA, Zhang B, Anbalgam K, Thomas T, Yang M (2018) Synthesis and application of nano-structured metal nitrides and carbides: a review. Prog Solid State Chem 50:1-15

2. Patsalas $P$, Kalfagiannis N, Kassavetis $S$, Abadias G, Bellas D, Lekka C, Lidorikis E (2018) Conductive nitrides: growth principles, optical and electronic properties, and their perspectives in photonics and plasmonics. Mater Sci Eng: R: Rep 123:1-55. https://doi. org/10.1016/j.mser.2017.11.001

3. Saha B, Shakouri A, Sands TD (2018) Rocksalt nitride metal/ semiconductor superlattices: a new class of artificially structured materials. Appl Phys Rev 5(2):021101. https://doi. org/10.1063/1.5011972

4. Ningthoujam R (2017) Chapter 10-synthesis and characterization of borides, carbides, and nitrides and their applications. In: Tyagi A, Banerjee S (eds) Materials under extreme conditions. Elsevier, Amsterdam, pp 337-375. https://doi.org/10.1016/B9780-12-801300-7.00010-3

5. Zhong Y, Xia X, Shi F, Zhan J, Tu J, Fan HJ (2016) Transition metal carbides and nitrides in energy storage and conversion. Adv Sci 3:1500286. https://doi.org/10.1002/advs.201500286

6. Suzuki K, Kaneko T, Yoshida H, Morita H, Fujimori H (1995) Crystal structure and magnetic properties of the compound $\mathrm{CoN}$. J Alloys Compd 224:232-236. https://doi.org/10.1016/09258388(95)01561-2

7. Oda K, Yoshio T, Oda K (1987) Preparation of Co-N films by rfsputtering. J Mater Sci 22:2729-2733. https://doi.org/10.1007/ BF01086464

8. Fang JS, Yang LC, Hsu CS, Chen GS, Lin YW, Chen GS (2004) Phase transition behavior of reactive sputtering deposited $\mathrm{Co}-\mathrm{N}$ thin films using transmission electron microscopy. J Vac Sci Technol A 22:698-704. https://doi.org/10.1116/1.1722656

9. Cruz WDL, Contreras O, Soto G, Perez-Tijerina E (2006) Cobalt nitride films produced by reactive pulsed laser deposition. REVISTAMEXICANA DE FíSICA 52:409-412

10. Das B, Reddy MV, Rao GVS, Chowdari BVR (2012) Synthesis of porous-CoN nanoparticles and their application as a high capacity anode for lithium-ion batteries. J Mater Chem 22:1750517510. https://doi.org/10.1039/C2JM31969A

11. Das B, Reddy MV, Chowdari BVR (2013) X-ray absorption spectroscopy and energy storage of ni-doped cobalt nitride, $\left(\mathrm{Ni}_{0.33}\right.$ $\left.\mathrm{Co}_{0.67}\right) \mathrm{N}$, prepared by a simple synthesis route. Nanoscale 5:1961-1966. https://doi.org/10.1039/C2NR33675H

12. Das B, Behm M, Lindbergh G, Reddy M, Chowdari B (2015) High performance metal nitrides, $\mathrm{MN}(\mathrm{M}=\mathrm{Cr}, \mathrm{Co})$ nanoparticles for non-aqueous hybrid supercapacitors. Adv Powder Technol 26:783-788. https://doi.org/10.1016/j.apt.2015.02.001

13. Wang Y, Zhang B, Pan W, Ma H, Zhang J (2017) 3d porous nickel cobalt nitrides supported on nickel foam as efficient electrocatalysts for overall water splitting. CHEMSUSCHEM 10:4170-4177. https://doi.org/10.1002/cssc.201701456

14. Zhang Y, Ouyang B, XU J, Jia G, Chen S, Rawat RS, Fan HJ (2016) Rapid synthesis of cobalt nitride nanowires: highly efficient and low-cost catalysts for oxygen evolution. Angew Chemie Int Ed 55:8670-8674. https://doi.org/10.1002/anie.201604372

15. Peng X, Pi C, Zhang X, Li S, Huo K, Chu PK (2019) Recent progress of transition metal nitrides for efficient electrocatalytic water splitting. Sustain Energy Fuels 3:366-381. https://doi. org/10.1039/C8SE00525G

16. Chen Z, Duan X, Wei W, Wang S, Ni BJ (2019) Recent advances in transition metal-based electrocatalysts for alkaline hydrogen evolution. J Mater Chem A 7:14971-15005. https://doi. org/10.1039/C9TA03220G

17. Wu Z, Nie D, Song M, Jiao T, Fu G, Liu X (2019) Facile synthesis of Co-Fe-B-P nanochains as an efficient bifunctional electrocatalyst for overall water-splitting. Nanoscale 11:7506-7512. https ://doi.org/10.1039/C9NR01794A

18. Kang JS, Kim JY, Yoon J, Kim J, Yang J, Chung DY, Mc Kim, Jeong H, Son YJ, Kim BG, Jeong J, Hyeon T, Choi M, Ko MJ, Sung YE (2018) Room-temperature vapor deposition of cobalt nitride nanofilms for mesoscopic and perovskite solar cells. Adv Energy Mater 8:1703114. https://doi.org/10.1002/aenm.201703114

19. Schmitz-Dumont O, Kron N (1955) Be1 thermischem abbau von kobalt(II1)-amid entstehende kobaltnitride. Angew Chemie 67:231

20. Liu X, Lu H, He M, Jin K, Yang G, Ni H, Zhao K (2014) The preparation and antiferromagnetic properties of epitaxial rocksalt-type CoN films. J Alloys Compd 582:75-78. https://doi.org/10.1016/j. jallcom.2013.08.001

21. Lukashev P, Lambrecht WRL (2004) First-principles study of the preference for zinc-blende or rocksalt structures in FeN and CoN. Phys Rev B 70:245205. https://doi.org/10.1103/PhysR evB.70.245205

22. Amudhavalli A, Manikandan M, Cinthia AJ, Rajeswarapalanichamy R, lyakutti K (2018) Structural, electronic, and mechanical properties of $\mathrm{CoN}$ and $\mathrm{NiN}$ : an ab initio study. Zeitschrift für Naturforschung A 72:321-330. https://doi.org/10.1515/ zna-2016-0377

23. Taylor B, Joyner B, Verhoek F (1961) Products of the thermal decomposition of some cobalt ammine azides. J Am Chem Soc 83:1069

24. Zhao X, Wang CZ, Yao Y, Ho KM (2016) Large magnetic anisotropy predicted for rare-earth-free $\mathrm{Fe}_{16-x} \mathrm{Co}_{x} \mathrm{~N}_{2}$ alloys. Phys Rev B 94:224424

25. Kumar A, Thakur PR, Sharma G, Naushad M, Rana A, Mola GT, Stadler FJ (2019) Carbon nitride, metal nitrides, phosphides, chalcogenides, perovskites and carbides nanophotocatalysts for environmental applications. Environ Chem Lett 17:655-682

26. Chen P, Xu K, Tong Y, Li X, Tao S, Fang Z, Chu W, Wu X, Wu C (2016) Cobalt nitrides as a class of metallic electrocatalysts for the oxygen evolution reaction. Inorg Chem Front 3:236-242

27. Fan Q, Sang L, Jiang D, Yang L, Zhang H, Chen Q, Liu Z (2019) Plasma enhanced atomic layer deposition of cobalt nitride with cobalt amidinate. J Vac Sci Technol A: Vac Surfaces Films 37(1):010904

28. Fang JS, Yang LC, Hsu CS, Chen GS, Lin YW, Chen GS (2004) Phase transition behavior of reactive sputtering deposited $\mathrm{Co}-\mathrm{N}$ thin films using transmission electron microscopy. J Vac Sci Technol A 22:698

29. Widenmeyer M, Shlyk L, Becker N, Dronskowski R, Meissner E, Niewa R (2016) Synthesis of metastable $\mathrm{Co}_{4} \mathrm{~N}, \mathrm{Co}_{3} \mathrm{~N}, \mathrm{Co}_{2} \mathrm{~N}$, and $\mathrm{CoO}_{0.74} \mathrm{~N}_{0.24}$ from a single azide precursor and intermediates in $\mathrm{CoBr}_{2}$ ammonolysis. Eur J Inorg Chem 2016:4792-4801

30. Niwa K, Terabe T, Kato D, Takayama S, Kato M, Soda K, Hasegawa M (2017) Highly coordinated iron and cobalt nitrides synthesized at high pressures and high temperatures. Inorg Chem 56:6410-6418

31. Stampfl C, Mannstadt W, Asahi R, Freeman AJ (2001) Electronic structure and physical properties of early transition 
metal mononitrides: density-functional theory LDA, GGA, and screened-exchange LDA FLAPW calculations. Phys Rev B 63:155106. https://doi.org/10.1103/PhysRevB.63.155106

32. Bhattacharyya $S$ (2015) Iron nitride family at reduced dimensions: a review of their synthesis protocols and structural and magnetic properties. J Phys Chem C 119:1601-1622. https://doi. org/10.1021/jp510606z

33. Li S, Cui X, Liu ZF, Song TL (2019) Phase transition, magnetic and electronic properties of iron mononitride: First-principles calculations. J Alloys Compd 771:322-326

34. Jouanny I, Weisbecker $P$, Demange V, Grafouté $M$, Peña O, BauerGrosse E (2010) Structural characterization of sputtered singlephase iron nitride coatings. Thin Solid Films 518:1883-1891. https://doi.org/10.1016/j.tsf.2009.07.039

35. Pak J, Lin W, Wang K, Chinchore A, Shi M, Ingram DC, Smith AR, Sun K, Lucy JM, Hauser AJ, Yang FY (2010) Growth of epitaxial iron nitride ultrathin film on zinc-blende gallium nitride. J Vac Sci Technol A 28:536-540. https://doi.org/10.1116/1.3425805

36. Gupta M, Tayal A, Gupta A, Reddy V, Horisberger M, Stahn J (2011) Study of non-magnetic iron mononitride thin films. J Alloys Compd 509:8283-8288. https://doi.org/10.1016/j.jallc om.2011.04.139

37. De-chun $\mathrm{H}$, Yong $\mathrm{P}$, Su-yuan L, Yong-lin H (2016) First-principles calculations on phase transition and elastic properties of CoN. Acta Physics Polonica A 130:743-747. https://doi.org/10.12693 /APhysPolA.130.743

38. Hlynsson VF, Skúlason E, Garden AL (2014) A systematic, firstprinciples study of the structural preference and magnetic properties of mononitrides of the d-block metals. J Alloys Compd 603:172-179. https://doi.org/10.1016/j.jallcom.2014.02.153

39. Soni HR, Mankad V, Gupta SK, Jha PK (2012) A first principles calculations of structural, electronic, magnetic and dynamical properties of mononitrides FeN and CoN. J Alloys Compd 522:106-113. https://doi.org/10.1016/j.jallcom.2012.01.100

40. Paduani C (2008) Band structure of the mononitrides CoN, $\mathrm{NiN}$ and $\mathrm{CuN}$ in the zincblende structure. Solid State Commun 148:297-300. https://doi.org/10.1016/j.ssc.2008.09.010

41. Gupta R, Pandey N, Tayal A, Gupta M (2015) Phase formation, thermal stability and magnetic moment of cobalt nitride thin films. AIP Adv 5:097131. https://doi.org/10.1063/1.4930977

42. Bunker $\mathrm{G}$ (2010) Introduction to XAFS: a practical guide to X-ray absorption fine structure spectroscopy. Cambridge University Press, Cambridge

43. Pandey N, Gupta M, Gupta R, Hussain Z, Reddy VR, Phase DM, Stahn J (2019) Structural and magnetic properties of stoichiometric $\mathrm{Co}_{4} \mathrm{~N}$ epitaxial thin films. Phys Rev B 99:214109. https:// doi.org/10.1103/PhysRevB.99.214109

44. Kumar Y, Pandey N, Gupta M (2019) Study of cobalt mononitride thin films deposited using different sized magnetron sources and effect of carbon doping. AIP Conf Proc 2115:030294. https ://doi.org/10.1063/1.5113133

45. Phase DM, Gupta M, Potdar S, Behera L, Sah R, Gupta A (2014) Development of soft x-ray polarized light beamline on Indus-2 synchrotron radiation source. AIP Conf Proc 1591:685-686. https ://doi.org/10.1063/1.4872719

46. Caliebe WA, Murzin V, Kalinko A, Görlitz M (2019) High-flux XAFSbeamline P64 at Petra III. AIP Conf Proc 2054:060031. https://doi. org/10.1063/1.5084662

47. Teo B, Joy D (1981) EXAFS spectroscopy: techniques and applications. Springer, Berlin. https://doi. org/10.1007/978-1-4757-1238-4

48. Conradson SD, Durakiewicz T, Espinosa-Faller FJ, An YQ, Andersson DA, Bishop AR, Boland KS, Bradley JA, Byler DD, Clark DL, Conradson DR, Conradson LL, Costello AL, Hess NJ, Lander GH, Llobet A, Martucci MB, Mustre de Leon J, Nordlund D, LezamaPacheco JS, Proffen TE, Rodriguez G, Schwarz DE, Seidler GT,
Taylor AJ, Trugman SA, Tyson TA, Valdez JA (2013) Possible bose-condensate behavior in a quantum phase originating in a collective excitation in the chemically and optically doped mott-hubbard system $\cup_{2+x}$. Phys Rev B 88:115135. https:// doi.org/10.1103/PhysRevB.88.115135

49. Rehr JJ, Kas JJ, Vila FD, Prange MP, Jorissen K (2010) Parameterfree calculations of $x$-ray spectra with FEFF9. Phys Chem Chem Phys 12:5503-5513. https://doi.org/10.1039/B926434E

50. Bunău O, Joly Y (2009) Self-consistent aspects of X-ray absorption calculations. J Phys Condens Matter 21:345501. https://doi. org/10.1088/0953-8984/21/34/345501

51. Stahn J, Glavic A (2016) Focusing neutron reflectometry: Implementation and experience on the ToF-reflectometer AMOR. Nucl Instrum Methods Phys Res Sect A: Accel Spectrom Detect Assoc Equip 821:44-54

52. Stahn J, Glavic A (2017) Efficient polarization analysis for focusing neutron instruments. J Phys Conf Ser 862:012007

53. Pandey N, Gupta M, Gupta R, Chakravarty S, Shukla N, Devishvili A (2017) Structural and magnetic properties of Co-N thin films deposited using magnetron sputtering at $523 \mathrm{~K}$. J Alloys Compd 694:1209-1213

54. Tessier F, Navrotsky A, Niewa R, Leineweber A, Jacobs H, Kikkawa S, Takahashi M, Kanamaru F, DiSalvo FJ (2000) Energetics of binary iron nitrides. Solid State Sci 2:457

55. Tripathi Y, Gupta R, Seema Gupta M, Phase D, Rajput P (2019) Study of phase formulation in CrN thin films and its response to a minuscule oxygen flow in reactive sputtering process. Thin Solid Films 670:113-121. https://doi.org/10.1016/j. tsf.2018.10.009

56. Hultman $L$ (2000) Thermal stability of nitride thin films. Vacuum 57:1-30. https://doi.org/10.1016/S0042-207X(00)00143-3

57. Zhao X, Ke L, Wang CZ, Ho KM (2016) Metastable cobalt nitride structures with high magnetic anisotropy for rare-earth free magnets. Phys Chem Chem Phys 18:31680-31690

58. Wang S, Yu X, Zhang J, Wang L, Leinenweber K, He D, Zhao Y (2016) Synthesis, hardness, and electronic properties of stoichiometric VN and CrN. Cryst Growth Des 16:351-358. https://doi. org/10.1021/acs.cgd.5b01312

59. Tayal A, Gupta M, Gupta A, Rajput P, Stahn J (2015) Origin of anomalous diffusion in iron mononitride thin films. Phys Rev $B$ 92:054109. https://doi.org/10.1103/PhysRevB.92.054109

60. Zhu L, Chen Z, Song $Y$, Wang $P$, Jiang $Y$, Jiang L, Zhou YN, $\mathrm{Hu} L$ (2018) Lower ammoniation activation energy of CoN nanosheets by $\mathrm{Mn}$ doping with superior energy storage performance for secondary ion batteries. Nanoscale 10:5581-5590. https://doi.org/10.1039/C7NR08893K

61. Soriano L, Abbate M, Pen H, Czyzyk M, Fuggle J (1993) The interaction of $\mathrm{N}$ with $\mathrm{Ti}$ and the oxidation of TiN studied by soft $\mathrm{x}$-ray absorption spectroscopy. J Electron Spectrosc Rel Phenomena 62(1):197-206. https://doi.org/10.1016/0368-2048(93)80015-E

62. Mitterbauer $C$, Hébert $C$, Kothleitner G, Hofer F, Schattschneider $\mathrm{P}$, Zandbergen $\mathrm{H}$ (2004) Electron energy loss-near edge structure as a fingerprint for identifying chromium nitrides. Solid State Commun 130:209-213. https://doi.org/10.1016/j. ssc.2004.01.045

63. Chen JG (1997) NEXAFS investigations of transition metal oxides, nitrides, carbides, sulfides and other interstitial compounds. Surface Sci Rep 30:1-152. https://doi.org/10.1016/S0167 -5729(97)00011-3

64. Papaconstantopoulos DA, Pickett WE, Klein BM, Boyer LL (1985) Electronic properties of transition-metal nitrides: The group-V and group-VI nitrides VN, NbN, TaN, CrN, MoN, and WN. Phys Rev B 31:752-761. https://doi.org/10.1103/PhysRevB.31.752

65. Rahman MM, Jiang ZT, Xie Z, Duan X, Zhou ZF, Wo PC, Yin CY, Mondinos N, Gu Q, Widjaja H, Jack K, Yago A, Amri A (2014) Understanding local bonding structures of $\mathrm{Ni}$-doped chromium 
nitride coatings through synchrotron radiation NEXAFS spectroscopy. J Phys Chem C 118:18573-18579. https://doi. org/10.1021/jp505004p

66. Kapoor R, Oyama ST, Friihberger B, DeVries BD, Chen JG (1995) Characterization of early transition metal carbides and nitrides by NEXAFS. Catal Lett 34:179-189. https://doi.org/10.1007/ BF00808333

67. Pflüger J, Fink J, Schwarz K (1985) Electronic structure of unoccupied states of stoichiometric $\mathrm{ZrN}, \mathrm{NbC}$ and $\mathrm{NbN}$ as determined by high energy electron energy loss spectroscopy. Solid State Commun 55:675-677. https://doi.org/10.1016/00381098(85)90231-5

68. Ankudinov A, Rehr J, Low J, Bare S (2002) Theoretical interpretation of XAFS and XANES in Pt clusters. Top Catal 18:3-7. https:// doi.org/10.1023/A:1013849814153

69. Dai Y, Gorey TJ, Anderson SL, Lee S, Lee S, Seifert S, Winans RE (2017) Inherent size effects on XANES of nanometer metal clusters: size-selected platinum clusters on silica. J Phys Chem C 121:361-374. https://doi.org/10.1021/acs.jpcc.6b10167

70. Bazin D, Sayers D, Rehr JJ, Mottet C (1997) Numerical simulation of the platinum $\mathrm{L}_{\text {/II }}$ edge white line relative to nanometer scale clusters. J Phys Chem B 101:5332-5336. https://doi.org/10.1021/ jp963949+

71. Frenkel Al, Yevick A, Cooper C, Vasic R (2011) Modeling the structure and composition of nanoparticles by extended $\mathrm{x}$-ray absorption fine-structure spectroscopy. Ann Rev Anal Chem 4:23-39. https://doi.org/10.1146/annurev-anchem-06101 0-113906

72. See electronic supplementary material describing results obtained by fitting the EXAFS data using different approaches

73. Fornasini P, Monti F, Sanson A (2001) On the cumulant analysis of EXAFS in crystalline solids. J Synchrotron Radiat 8:1214-1220. https://doi.org/10.1107/S0909049501014923
74. Yevick A, Frenkel Al (2010) Effects of surface disorder on exafs modeling of metallic clusters. Phys Rev B 81:115451. https://doi. org/10.1103/PhysRevB.81.115451

75. Bunker G (1983) Application of the ratio method of EXAFS analysis to disordered systems. Nucl Instrum Methods Phys Res 207:437-444. https://doi.org/10.1016/0167-5087(83)90655-5

76. Cheballah Y, Ziane A, Bouarab S, Vega A (2017) Density functional study of the optical response of FeN and $\mathrm{CoN}$ nitrides with zinc-blend and rock-salt structures. J Phys Chem Solids 100:148-153. https://doi.org/10.1016/j.jpcs.2016.09.016

77. Blundell SJ, Bland JAC (1992) Polarized neutron reflection as a probe of magnetic films and multilayers. Phys Rev B 46:33913400. https://doi.org/10.1103/PhysRevB.46.3391

78. Hope S, Lee J, Rosenbusch P, Lauhoff G, Bland JAC, Ercole A, Bucknall D, Penfold J, Lauter HJ, Lauter V, Cubitt R (1997) Thickness dependence of the total magnetic moment per atom in the $\mathrm{Cu} / \mathrm{Ni} / \mathrm{Cu} / \mathrm{Si}(001)$ system. Phys Rev B 55:11422-11431. https:// doi.org/10.1103/PhysRevB.55.11422

79. Cubitt R, Fragneto $G$ (2002) Chapter 2.8.3-neutron reflection: principles and examples of applications. In: Pike R, Sabatier P (eds) Scattering. Academic Press, London, pp 1198 - 1208. https ://doi.org/10.1016/B978-012613760-6/50065-6

80. Ott F SIMULREFLEC (V1.7 2011). http://www-Ilb.cea.fr/prism/ programs/simulreflec/simulreflec.html

Publisher's Note Springer Nature remains neutral with regard to jurisdictional claims in published maps and institutional affiliations. 